\title{
Balanced Scorecard (BSC) - uma visão metodológica para o acompanhamento de sua implementação
}

Revista do

Serviço

Público

Ano 54

Número 3

Jul-Set 2003

\author{
Edla M. B. Lima, Maristela J. da Silva, \\ Marlene Araújo e Cintia P. da Cunha
}

\section{Introdução}

A Empresa Brasileira de Pesquisa Agropecuária (Embrapa), vinculada ao Ministério da Agricultura, Pecuária e Abastecimento, vem utilizando o método de planejamento estratégico desde 1989, com vistas à elaboração de planos estratégicos, denominados internamente de Plano Diretor da Embrapa (PDE) (1999), escopo corporativo, e Plano Diretor da Unidade (PDU), escopo das unidades de negócio, chamadas de Unidades Descentralizadas (UDs). Na evolução do processo de planejamento estratégico, a empresa detectou a necessidade de adotar método efetivo de gestão do PDE (1999) e dos PDUs, visando a tirar a estratégia do papel e colocá-la em ação. Como conseqüência, a partir do segundo semestre de 1996, iniciaram-se os esforços de adaptação e implementação do método Balanced Scorecard (BSC), de Kaplan (1996), objetivando a implementação e operacionalização do PDE (1996) e dos PDUs, como também a internalização dos conceitos e a implementação do processo de gestão estratégica e a integração dos instrumentos de gestão existentes na empresa.

A adaptação do Balanced Scorecard para a Embrapa foi desenvolvida simultaneamente com a publicação do método por Kaplan (1996) e a partir de um projeto piloto executado em uma das unidades descentralizadas da Embrapa, Centro Nacional de Pesquisa em Agroindústria de Alimentos, em 1997. O resultado do projeto piloto consolida uma metodologia denominada Mogest (1998-1999), que foi adaptada à realidade de uma instituição pública e de pesquisa, a Embrapa, e deu origem ao Modelo de Gestão Estratégica (MGE). No período de 1999 a 2001, o

Edla Maria Bezerra Lima, MSc em Geologia de Engenharia e Ambiental; Maristela Jesus da Silva, BSc. em Ciências

Sociais;

Marlene de

Araújo, BSc, em

Comunicação

Social; e

Cintia Pereira

da Cunha, graduada em

Administração.

Todas são

servidoras da

Secretaria de

Gestão e

Estratégia

(SGE) da

Embrapa,

Brasília-DF.

Contato:

edla.lima@

embrapa.br

maristela.silva@ embrapa.br

marlene@

sede.embrapa.br cintia@

sede.embrapa.br 
MGE foi estendido a toda a empresa e, a partir de 2000, deu-se início ao processo de acompanhamento do MGE naquelas unidades modeladas na primeira e segunda fases, com a aplicação da metodologia denominada MGE/Acompanha (2000). O método aborda, no biênio 2000-2001, o acompanhamento do MGE por meio de questionários aplicados aos Gerentes de Objetivos Estratégicos (GOEs) e do uso de ferramentas para a gestão da informação, por meio dos softwares Same (1999), que é um banco de dados, WEBMGE (2000), que integra, executa e acompanha todos os MGEs em ambiente WEB, e Oracle BSC (2002), que executa o processo de avaliação (painel de bordo).

A partir do segundo semestre de 2002, inicia-se a terceira fase do MGE com a inserção deste no Sistema Integrado de Gestão (SEG), em fase de implementação.

\section{Construção e implementação do método BSC na Embrapa}

A construção e implementação do MGE da Embrapa teve início em agosto de 1996, como resultado do pós-doutorado de um pesquisador da empresa, que na época exercia suas atividades na Secretaria de Gestão e Estratégia (SGE), unidade central da administração responsável pela gestão estratégica. O resultado desse trabalho foi apresentado à Diretoria Executiva (DE), que, convencida dos benefícios que a ferramenta poderia trazer para medir o grau de execução da estratégia, o cumprimento da missão e o alcance da visão delineados no Plano Diretor, aprovou o projeto. Na impossibilidade de contratação de consultoria externa, com knowhow na construção, implementação e acompanhamento do método $B S C$, formou-se uma equipe (Equipe MGE) composta de técnicos da própria empresa, designada pela Diretoria Executiva, com o objetivo de executar um projeto piloto em uma das unidades da Embrapa, para teste e adaptação do método $B S C$. A primeira tarefa dessa equipe foi "vender" a idéia para um conjunto de oito unidades. A unidade candidata passaria pelo aprendizado de construir, em conjunto com a Equipe MGE, de forma participativa, um instrumento de gestão ajustado à sua cultura, valores e características, que possibilitaria o planejamento, execução, acompanhamento e avaliação das estratégias preconizadas em seu Plano Diretor da Unidade (PDU).

Em 1997, a Embrapa Agroindústria de Alimentos, unidade localizada no Rio de Janeiro, com 140 empregados, aderiu ao processo, aceitando funcionar como laboratório experimental para adaptar o método BSC aos valores, à cultura e a forma de organização do trabalho da empresa. $\mathrm{O}$ projeto piloto alcançou os resultados preconizados na proposta do MGE, com a obtenção dos seguintes resultados principais: 
a) aprendizado, internalização e adaptação do método $B S C$ às particularidades da Embrapa e de uma de suas UDs; e,

b) elaboração do MGE da Embrapa Agroindústria de Alimentos.

Simultaneamente à fase de implemantação do MGE da Embrapa Agroindústria de Alimentos, esse modelo começou a ser introduzido no restante da empresa, com a construção de MGEs para outras UDs, respeitadas as suas características peculiares. A metodologia de escolha dessas unidades obedeceu à divisão existente na empresa quanto à distinção das unidades, em razão da sua orientação de pesquisa (produtos e animais, temas e ecorregião). Foram escolhidas as seguintes unidades: Embrapa Semi-Árido (Petrolina/PE), Embrapa Gado de Leite (Juiz de Fora/MG) e Embrapa Soja (Londrina/PR). Juntamente com essas unidades, deu-se início ao projeto de construção do MGE Corporativo (central).

Tomada a decisão de implementar o projeto em toda a empresa, no período de 2000 a 2001, foi elaborada uma versão simplificada do método Mogest (Mogest Simplificado, 2000) e ampliada a Equipe MGE, que passou a contar com a colaboração de facilitadores metodológicos, técnicos das Unidades Descentralizadas com conhecimento em gestão, que foram treinados pela Equipe MGE, de forma a construir o MGE de sua unidade. Aqueles mais efetivos foram escolhidos para dar suporte à Equipe MGE, com o objetivo de acelerar a implementação do modelo naquelas unidades que apresentaram maior dificuldade. No final de 2001, das 40 unidades da empresa 38 já estavam com os seus modelos construídos e, em 2002, iniciou-se a fase de implementação, o que efetivamente representa a execução das estratégias estabelecidas no PDE (1999) e PDUs.

A premissa básica do processo de construção do MGE Corporativo e do MGE das UDs foi a busca de interação, comunicação e avaliação global e estratégica da performance da instituição como um todo, de forma a permitir a avaliação da capacidade da empresa em dar resposta aos diversos setores da sociedade e, especialmente, mensurar os impactos sociais, econômicos e ambientais provocados pelos resultados gerados. A implementação de modelo de gestão capaz de atingir esse propósito necessitou contar com o entendimento e comprometimento das pessoas envolvidas com as atividades de gestão. Assim, a preocupação maior da Equipe MGE foi estabelecer uma relação de confiança, transparência e participação, utilizando-se, no decorrer do processo, de recursos de valorização das intenções e ações dos empregados, por meio de entrevistas e coleta de informações a respeito das dificuldades existentes para a execução do projeto, dos pontos de estrangulamentos organizacionais, operacionais e individuais e das vantagens e desvantagens competitivas para a consecução dos planos estratégicos. Essa atividade foi desenvolvida na forma de oficinas de trabalho. 
O produto resultante das oficinas de trabalho, após validação pela Diretoria Executiva (DE) e pelo Conselho de Administração (Consad), foi submetido à apreciação das unidades para conhecimento, informação e sugestões, com vistas à melhoria do modelo. O resultado desse processo foi a elaboração do MGE Corporativo (Figura 1), que gerou a construção do Plano de Ação Estratégica (PAE) (Figura 2), cujo objetivo é organizar e conduzir a empresa de forma estratégica, partindo do estado atual para o estado futuro desejado, explicitado no Plano Diretor (1999), em especial no que se refere à missão, à visão e às diretrizes estratégicas.

\section{Modelo de Gestão Estratégica Corporatno da Embrapa}

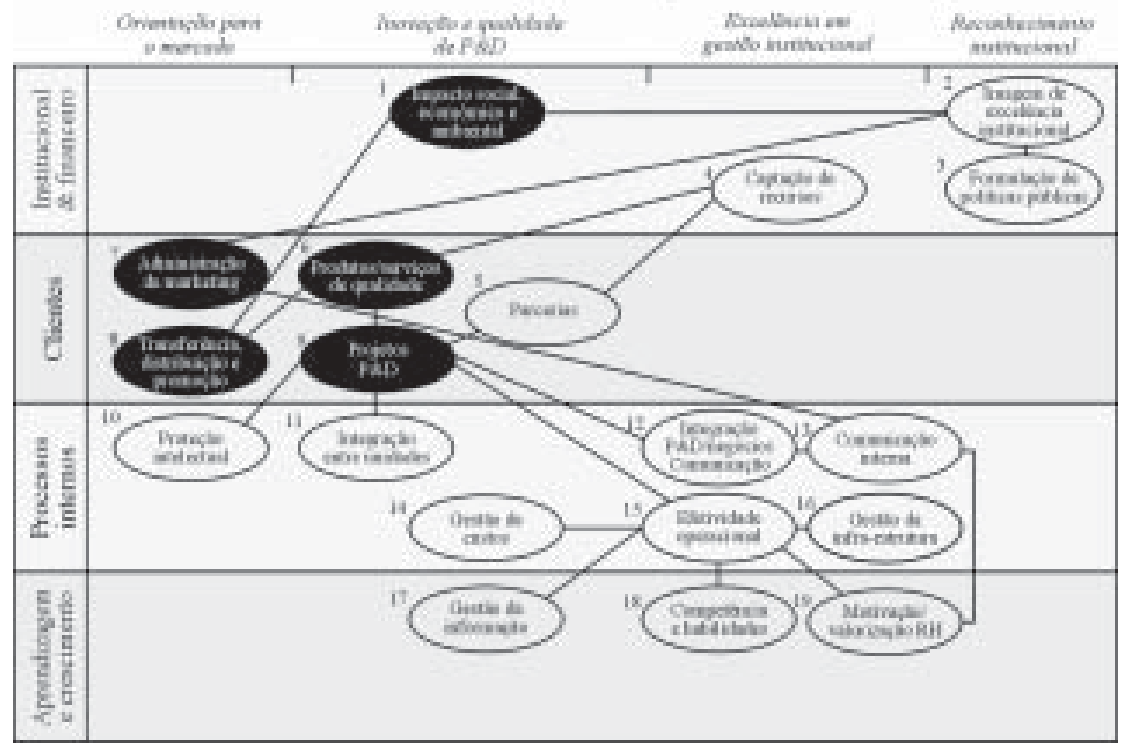

Figura 1: Diagrama do MGE Corporativo com destaque para os objetivos estratégicos comuns a toda a empresa. 


\begin{tabular}{|c|c|c|c|c|}
\hline \multicolumn{5}{|c|}{$\begin{array}{l}\text { Modelo de Gestão Estratégica - Embrapa } \\
\text { Ano de trabalho: } 2001\end{array}$} \\
\hline \multicolumn{5}{|c|}{ Plano de ações estratégicas por objetivo estratégico } \\
\hline \multicolumn{5}{|c|}{$\begin{array}{l}\text { Objetivo estratégico: } 6 \text { - Gerar e adaptar produtos e serviços de qualidade } \\
\text { Gerentes: XXXXXXXXXXXXXXXX }\end{array}$} \\
\hline \multicolumn{5}{|c|}{ Iniciativa: 1. Aprimorar a gestão de processos para a melhoria contínua de P\&D } \\
\hline № da ação & 6.1 .1 & 6.1 .2 & 6.1 .3 & 6.1 .4 \\
\hline $\begin{array}{l}\text { Ação pre- } \\
\text { vista para o } \\
\text { período }\end{array}$ & $\begin{array}{l}\text { Promover o } \\
\text { alinhamento das } \\
\text { metas do SEP } \\
\text { com o PPA }\end{array}$ & $\begin{array}{l}\text { Promover } \\
\text { análise das impli- } \\
\text { cações e desdo- } \\
\text { bramentos que o } \\
\text { PPA terá sobre o } \\
\text { SEP }\end{array}$ & $\begin{array}{l}\text { Análise crítica da } \\
\text { experiência do } \\
\text { SEP após o } \\
\text { primeiro ciclo } \\
\text { (1994-2000), } \\
\text { visando ao seu } \\
\text { aprimoramento }\end{array}$ & $\begin{array}{l}\text { Análise crítica da } \\
\text { experiência do } \\
\text { modus operandi } \\
\text { dos CTIs e CTPs, } \\
\text { visando à melho- } \\
\text { ria contínua da } \\
\text { qualidade da pro- } \\
\text { gramação de } \\
\text { P\&D }\end{array}$ \\
\hline Responsável & XXXX & XXXX & XXXX & XXXX \\
\hline $\begin{array}{l}\text { OEs } \\
\text { relacionados }\end{array}$ & $2,15,17$ & $2,15,17$ & $\begin{array}{l}2,8,9,12,13 \\
14,15,17\end{array}$ & $11,13,15,19$ \\
\hline \multicolumn{5}{|l|}{ Prioridade } \\
\hline \multicolumn{5}{|l|}{ NIA } \\
\hline Prazo & $31 / 12 / 2000$ & $31 / 12 / 200$ & $31 / 12 / 2000$ & $31 / 12 / 2000$ \\
\hline $\begin{array}{l}\text { Estratégia de } \\
\text { implantação }\end{array}$ & $\begin{array}{l}\text { 1. Mapear a } \\
\text { relação entre as } \\
\text { metas dos pro- } \\
\text { gramas do PPA } \\
\text { e do SEP }\end{array}$ & $\begin{array}{l}\text { 1. Com base no } \\
\text { documento de } \\
\text { alinhamento, } \\
\text { negociar, inter- } \\
\text { namente, com os } \\
\text { segmentos } \\
\text { envolvidos da } \\
\text { empresa a gestão } \\
\text { dos objetivos } \\
\text { maiores do PPA } \\
\text { e do SEP }\end{array}$ & $\begin{array}{l}\text { 1. Utilizando } \\
\text { metodologia } \\
\text { participativa, } \\
\text { comparar } \\
\text { fundamentos } \\
\text { conceituais do } \\
\text { SEP com a } \\
\text { experiência } \\
\text { verificada nos } \\
\text { últimos cinco } \\
\text { anos, identificar } \\
\text { gargalos e propor } \\
\text { aprimoramento } \\
\text { para o desem- } \\
\text { penho de gestão }\end{array}$ & $\begin{array}{l}\text { 1. Associada à } \\
\text { ação anterior, } \\
\text { utilizando-se } \\
\text { metodologia } \\
\text { participativa e } \\
\text { técnicas de } \\
\text { análise de pro- } \\
\text { cesso, analisar a } \\
\text { operação desses } \\
\text { mecanismos de } \\
\text { articulação, } \\
\text { identificando } \\
\text { pontos de } \\
\text { estrangulamento } \\
\text { e propor apri- } \\
\text { moramento para } \\
\text { o desempenho de } \\
\text { gestão }\end{array}$ \\
\hline $\begin{array}{l}\text { Resultados } \\
\text { esperados }\end{array}$ & $\begin{array}{l}\text { 1. Documento } \\
\text { com o alinha- } \\
\text { mento das metas } \\
\text { elaboradas }\end{array}$ & $\begin{array}{l}\text { 1. Documento } \\
\text { com proposição } \\
\text { negociada }\end{array}$ & $\begin{array}{l}\text { 1. Documento } \\
\text { com diretrizes } \\
\text { para aprimora- } \\
\text { mento geral do } \\
\text { SEP atendendo } \\
\text { os princípios de } \\
\text { melhoria na } \\
\text { geração e adap- } \\
\text { tação de produ- } \\
\text { tos e serviços }\end{array}$ & $\begin{array}{l}\text { 1. Documento } \\
\text { indicador que } \\
\text { subsidiará o } \\
\text { produto de ação } \\
\text { anterior. }\end{array}$ \\
\hline
\end{tabular}

Figura 2: Plano de ação estratégico do objetivo estratégico 6 do MGE Corporativo. 
O MGE Corporativo foi construído a partir do PDE (1999) por meio da análise e do detalhamento dos seus componentes: missão, visão, objetivos globais, diretrizes e projetos estruturantes.

Da decomposição da visão constante no PDE (1999), foram criados quatro temas estratégicos, a saber:

- orientação para o mercado;

- inovação e qualidade de P\&D;

- excelência em gestão institucional;

- reconhecimento institucional.

A partir desses temas estratégicos, foram definidos os objetivos estratégicos (OEs).

O método Balanced Scorecard preconiza e estrutura-se em quatro perspectivas organizacionais, que são: financeira; clientes; processos internos e aprendizado e crescimento, que serão permanentes durante todo o ciclo de vida da empresa. Nelas foram alocados os objetivos estratégicos, visando ao desenvolvimento equilibrado (por isso o termo "balanced”) da empresa. É importante mencionar que, no método de construção do MGE, os objetivos estratégicos foram alocados na matriz formada pelos temas estratégicos (colunas) e perspectivas organizacionais (linhas), formando o diagrama do MGE, composto por 19 OEs.

Nos MGEs das unidades descentralizadas, foram mantidos os mesmos temas estratégicos, perspectivas organizacionais e cinco objetivos estratégicos comuns (Figura 1, parte colorida) constantes do MGE Corporativo, com o objetivo de manter a coerência de conteúdo do Plano Diretor e do método Mogest.

Algumas unidades descentralizadas formularam estratégias específicas e pertinentes aos seus negócios. Além dos objetivos estratégicos comuns ao MGE Corporativo, foram elaborados os planos de ação estratégica para cada objetivo estratégico (Figura 2) e definidos os resultados a serem alcançados, observando-se a compatibilidade com outros instrumentos de planejamento em uso na empresa, como, por exemplo, o Plano Anual de Trabalho (PAT).

\section{O acompanhamento - método MGE/Acompanha}

O processo de acompanhamento do MGE efetua-se por meio de duas atividades básicas: 1) gestão da informação (base de dados, acompanhamento e avaliação) do modelo por meio do desenvolvimento dos softwares Sistema de Apoio a Modelagem Estratégica - SAME (1999), WEBMGE (2000) e Oracle BSC - OBSC (2002), modelados por técnicos da Embrapa e; 2) realização de oficinas de acompanhamento, em 2000, 
com a elaboração da primeira versão de um método para acompanhamento do MGE (Corporativo e UDs), contendo questionários para a avaliação da efetividade/eficiência do método. O segundo procedimento metodológico foi aplicado no acompanhamento do MGE Corporativo e de 12 UDs modeladas na primeira fase de construção/implementação do modelo, a saber: Embrapa Agroindústria de Alimentos (Guaratiba - RJ), Embrapa Agroindústria Tropical (Fortaleza - CE), Embrapa Agropecuária Oeste (Dourados - MS), Embrapa Gado de Leite (Juiz de Fora - MG), Embrapa Informática Agropecuária (São Carlos - SP), Embrapa Meio Ambiente (Jaguariúna - SP), Embrapa Meio Norte (Teresina - PI), Embrapa Pantanal (Corumbá - MS), Embrapa Rondônia (Porto Velho RO), Embrapa Semi-Árido (Petrolina - PE), Embrapa Solos (Rio de Janeiro - RJ), Serviço de Negócios para Transferência de Tecnologia (Brasília - DF), Embrapa Soja (Londrina - PR) e Embrapa Sede (Brasília - DF). Em 2002, simultaneamente ao acompanhamento dos MGEs da fase 1 , inicia-se a fase 2 , que consiste na construção/implementação do MGE nas demais UDs.

O método de acompanhamento denominado internamente de MGE/ Acompanha (2000) e executado no período de 2000 a 2001, em 13 unidades da empresa, consistiu na aplicação de um manual orientador composto de formulários (anexos), elaborados com o objetivo de avaliar os seguintes atributos no processo de implementação do MGE: motivação, envolvimento/comprometimento dos gerentes, equipes e empregados, divulgação e comunicação do MGE nas unidades, implementação efetiva do MGE (indicadores de desempenho e alvos de performance), integração com os outros instrumentos gerenciais da empresa e nível de desenvolvimento e capacitação dos empregados em gestão estratégica. O MGE/ Acompanha possibilitou o acompanhamento da implementação do MGE daquelas unidades da primeira fase, permitindo avaliar o grau de internalização e funcionamento do modelo e as respectivas dissonâncias culturais. A partir desse conhecimento, foi possível introduzir melhorias no processo de gestão estratégica da empresa.

Em 2002, introduzem-se ao Método MGE/Acompanha os diagramas apresentados no Método de Acompanhamento para uma Organização focada na estratégia, de Norton (2001), apud Coutinho (2002), na Figura 3, de forma adaptada à realidade do MGE da Embrapa, figuras 4,5 e 6 . 
Processo de mudança, que pode levar de 2 a 4 anos.

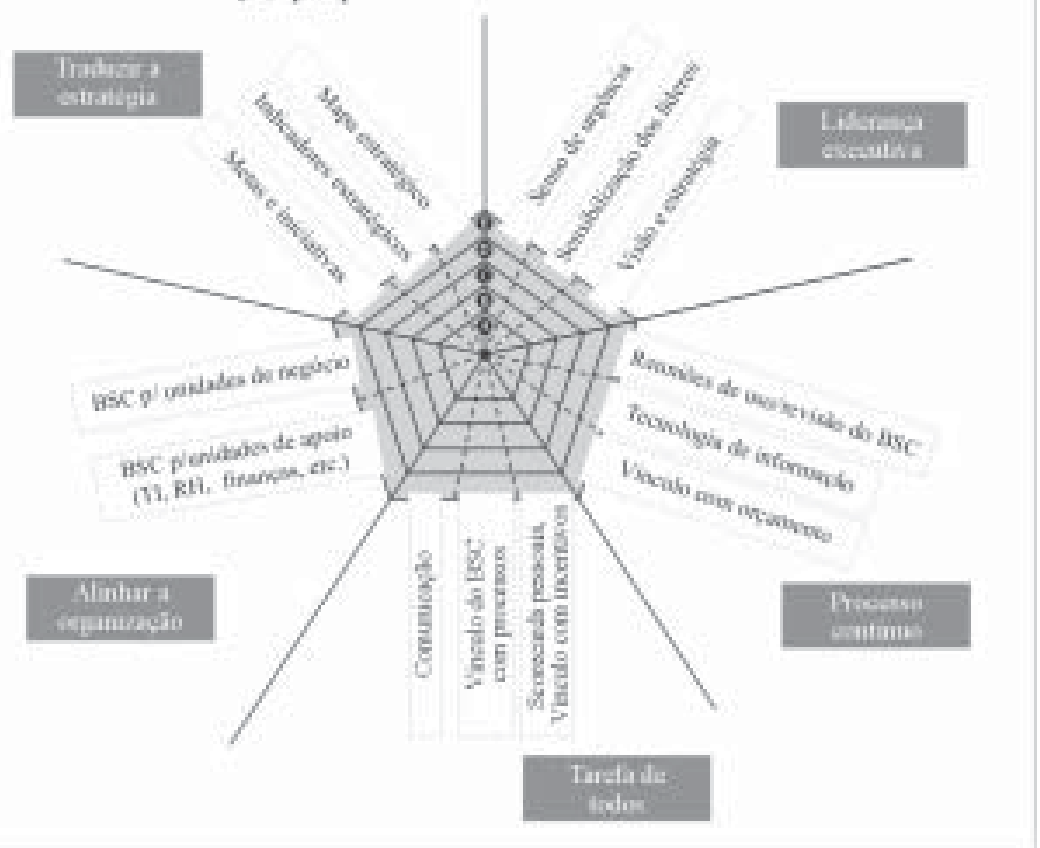

Figura 3: Diagrama de acompanhamento de Norton, apud Coutinho (2002). 


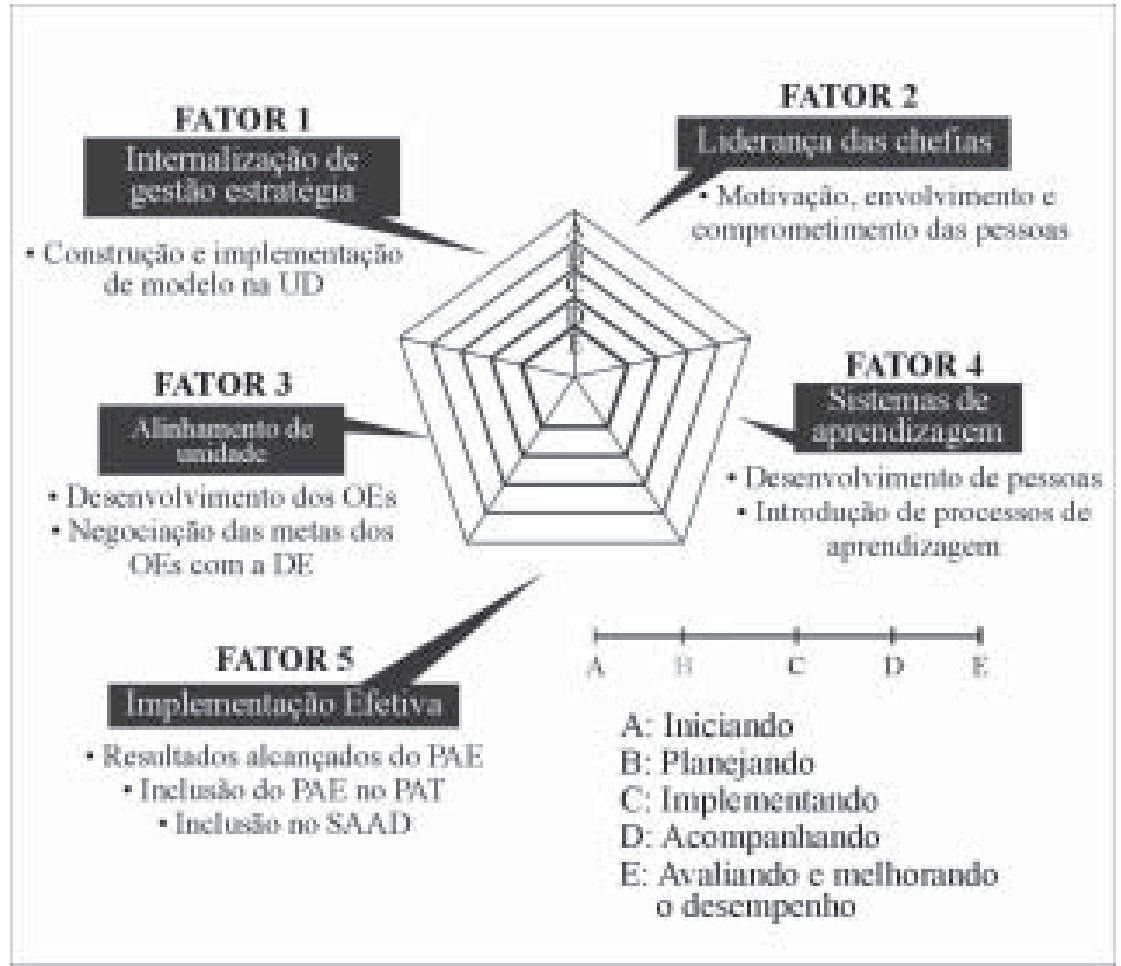

Figura 4: Diagrama adaptado de Norton, apud Coutinho (2002), para acompanhamento da implementação do MGE da UD. 


\section{Acompanhamento de execução dos OEs do MGE da UD X}

Temas de diagrama

- Orientaçio para o mercale

- Inevaçào e qualidade em P\&D

- Reconhecimento institucional

- Excelíncia em gestīo institucienal

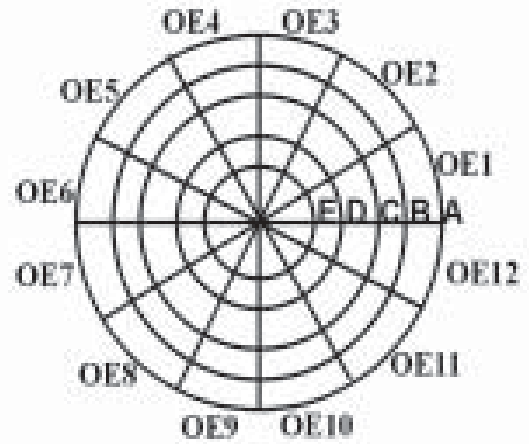

E- 2eso rralisade ou apreximad anconte

D) $40 \%$ realieado su aprosimadamente

C; $60 \% 5$ realicade su apersimadamente

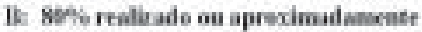

Ai foefs realirade on apreximad ameste

Figura 5: Diagrama para acompanhamento da implementação do MGE da UD, mostrando a distribuição dos OEs por temas estratégicos do PDE.

Acompanhamento de execução dos OEs do MGE da UD X

Ex.: $N^{*}$ e descrição do objetivo estratégico

Isiciativa $\mathrm{I}:$ (Descriçüs da iniciariva $)=\%$ do esecutada

Iniciativ a $\mathrm{N}$ : (Descriço du imiciafiox $)=\%$ do executado

Iniciativa $\mathrm{I}: \mathrm{XX}=45^{5}$.

Iniciativa $2: 17=67 \%$,

Imiciarive $N(\beta): Z Z=80^{\circ}$,

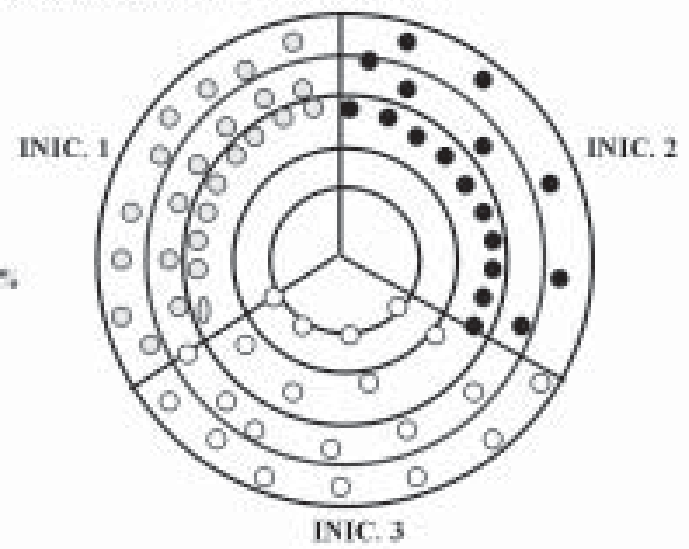

Figura 6: Diagrama para acompanhamento da implementação do OE do MGE da UD, mostrando a percentagem de realização das iniciativas estratégicas do $\mathrm{OE}$. 


\section{Resultados}

A aplicação do Método MGE/Acompanha nas UDs modeladas, na primeira fase de construção/implementação do modelo, biênio 2000-2001, proporcionou uma avaliação do MGE a partir das respostas dadas aos questionários (anexos) pelos gerentes de objetivos estratégicos e gerentes de modelos. Os dados foram compilados e analisados pela Equipe MGE, cujas informações mais relevantes são mostradas a seguir.

A efetiva implementação dos objetivos estratégicos e o devido controle da coerência e integração das ações estratégicas são parte de processo complexo de gestão, que exige comprometimento individual e habilidade para trabalho em grupo, objetivando a consecução dos alvos institucionais propostos.

O processo de administração estratégica requer novas maneiras de organização do trabalho e, consequientemente, novas atitudes pessoais, com vistas a uma visão global das diretrizes e metas institucionais. Isso implica, como em qualquer processo de mudança, utilização de meios e ferramentas que possam atuar sobre o comportamento das pessoas para, a partir disso, operar mudanças na cultura e ambiente organizacionais. Conscientes dessas premissas, a Equipe MGE não se limitou a trabalhar o processo apenas no campo das atividades, mas também se preocupou em atuar no campo do comportamento e da cultura da organização, entendendo o processo como de mudança social que necessita de tempo para ser assimilado, internalizado e consolidado.

No decorrer do processo de mudança social, foi possível observar barreiras de diversas ordens: cultural, administrativa/normativa, de política organizacional, de habilidade e competência, de comunicação e de aprendizado, etc. A aplicação do método MGE/Acompanha permitiu mapear os fatores críticos e inovadores para o sucesso da execução do modelo e, a partir daí, traçar um plano de trabalho para os próximos anos.

\section{Fatores críticos identificados}

a) Barreiras administrativa/normativas - Inconsistência das normas administrativas que apontam direções diferentes, exigem os mesmos recursos e não definem prioridades, como também provocam acúmulo de obrigações para os empregados, causando falta de entendimento de quais são, de fato, os direcionamentos estratégicos. Esse quadro, adicionado ao baixo nível de autonomia e autogestão, provoca nas pessoas uma sensação de impotência e inércia. 
b) Barreiras relacionadas às competências e habilidades - Nível inadequado de profissionalização em gestão das pessoas que ocupam posições de gerência e de tomada de decisão, diante das exigências, cada vez maiores, de um mercado competitivo, que requer competência e habilidade por parte dos líderes de negócios. Paradoxo em relação à alta qualificação dos profissionais da área fim, comparativamente aos da área meio. Estratégia inadequada quanto à indicação de profissionais pesquisadores sem experiência em gestão para ocupar cargos administrativos.

c) Barreiras relacionadas a comunicação e aprendizado Carência de profissionais capacitados em administração estratégica, o que resulta na dificuldade de disseminação de novos conceitos e métodos relacionados ao tema. A utilização de facilitadores metodológicos foi uma tentativa de minimizar essa carência, mas é preciso ressaltar que os facilitadores são voluntários das mais variadas especialidades, que, por mais boa vontade, possuem um grau de limitação teórica e prática para o exercício da atividade. O tema é complexo para ser assimilado em curto prazo e de maneira uniforme. Esse desnivelamento no processo de assimilação provoca desconforto em alguns e expectativas exageradas em outros, promovendo um clima de dúvidas e sensação de ambiente instável.

d) Barreiras políticas e culturais - $\mathrm{O}$ ambiente organizacional foi, por muito tempo, voltado para si mesmo, com baixa integração com o ambiente externo, provocando um comportamento de isolamento e reforçando nos empregados a falsa idéia de que a organização existe para si mesma. Isso dificulta o entendimento das pessoas quanto à importância do planejamento e da execução de estratégias que visem ao atendimento das demandas da sociedade, às exigências do mercado, à flexibilização da estrutura organizacional e à melhoria do relacionamento com os diversos segmentos do ambiente externo.

\section{Fatores inovadores identificados}

a) Metodologia - Alteração da perspectiva organizacional financeira do método $B S C$ para institucional e financeira. Essa adaptação do método $B S C$ resolveu o problema de avaliar a performance de uma organização pública com base em aspectos puramente financeiros (lucro, ROI, etc.), passando a considerar o seu impacto na sociedade.

b) Integração - Utilização de processo de modelagem participativo para criação dos MGE, mobilizando grande número de empregados de várias unidades da empresa, independentemente de sua posição hierárquica.

c) indicador de desempenho - Utilização de métricas de desempenho (indicadores de desempenho e alvos de performance) intrinsecamente conectadas com as estratégias da organização, ou seja, com o conteúdo dos planos estratégicos (PDE e PDUs). Outra inovação 
relacionada aos indicadores de desempenho foi o aprendizado de gestão

estratégica por parte dos gerentes de UDs, gerentes de objetivos estratégicos e outros empregados envolvidos no processo de implementação, acompanhamento e avaliação do MGE.

d) Liderança - Ampliação da capacidade gerencial, decorrente da seleção de gerentes de objetivos estratégicos, que possibilitou a democratização do processo decisório, o qual era centralizado em típica estrutura organizacional verticalizada, passando a viabilizar processo mais horizontal, com maior agilidade e flexibilidade.

e) Comunicação - Processo permanente de comunicação durante a criação do MGE, com o propósito de reduzir barreiras culturais, promover a motivação para a mudança e estimular a participação dos empregados;

f) Gestão da informação - Desenvolvimento de três softwares para apoio na gestão da informação do projeto MGE: 1) Sistema de Apoio a Modelagem Estratégica (Same) - para apoio durante o processo de construção do MGE, possibilitando o registro das informações produzidas em dinâmicas de grupo, registro dos componentes do MGE e do respectivo PAE; 2) WEBMGE - para apoiar na execução, no acompanhamento e na avaliação do MGE, visando a coletar dados que demonstrem a consecução dos objetivos estratégicos, por meio da aferição dos indicadores de desempenho e iniciativas estratégicas associadas; e 3) Oracle BSC (OBSC) (protótipo) - para integrar e apoiar o acompanhamento e a avaliação dos componentes estratégicos (MGE) e os de produção (PAT).

Em 2002, a Equipe MGE introduz os diagramas de Norton, apud Coutinho (2002), de forma adaptada, no Método MGE/Acompanha, figuras 4, 5 e 6, testado em uma unidade de negócio, apresentando resultados efetivos quanto ao uso para o acompanhamento da implementação do MGE e a internalização do conceito de "painel de bordo", utilizado no software Oracle BSC (OBSC) para avaliação.

A análise do diagrama da Figura 7 revela que o processo de "construir e implementar o MGE - Fator 1" encontra-se mais internalizado e com maior facilidade de execução do que as "ações de negociar as metas com a Diretoria Executiva - Fator 3"; "envolver, capacitar e motivar as pessoas - Fatores 2 e 4"; e "incluir as atividades estratégicas no Plano de Trabalho Anual (PAT) da Unidade e no Plano de Trabalho dos Empregados (SAAD) - Fator 5". 


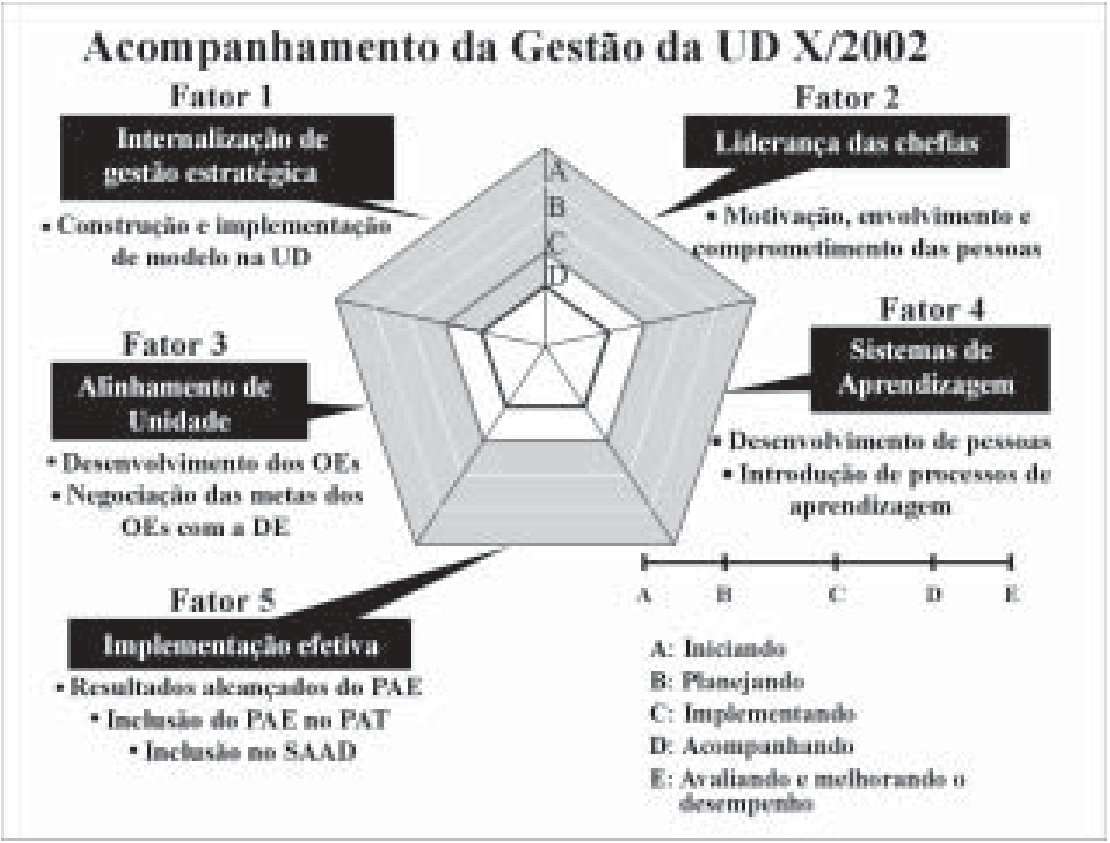

\section{Figura 7: Acompanhamento da implementação} do MGE da UD X.

A análise da implementação dos OEs da UD X/2002 (1- Implantar e gerir o processo de administração de marketing, melhorar a transferência de produtos, serviços e ampliar a captação de recursos; 2 - Desenvolver projetos de P\&D focados no mercado; 3 - gerar, adaptar e disponibilizar produtos/serviços de qualidade; 4 - Aperfeiçoar a gestão da informação técnico-centífica, de mercado e administrativa; 5 -Aprimorar o processo de capacitação de pessoas atendendo as necessidades da UD; 6 Promover a integração interinstitucional; 7 - Implantar e gerenciar o processo de comunicação interna; 8 - Promover a excelência operacional; 9 - Estabelecer mecanismos para integração do processo de P\&D com as demais áreas da unidade; 10 - Implantar mecanismos de motivação e de reconhecimento por resultados; 11 - Avaliar impactos ambientais e socioeconômicos; 12 - Construir imagem de centro de referência, por meio do diagrama representado na Figura 8, revela que, nessa unidade de negócio, os OEs relativos ao tema estratégico "Excelência em gestão" que abordam a gestão da informação, comunicação interna, excelência operacional, capacitação de pessoas e integração de P\&D com as demais áreas da UD encontram-se mais implementados do que os OEs que abordam a relação de motivação e reconhecimento por resultados, construção da imagem de centro de referência, avaliação de impactos ambientais e socioeconômicos, marketing, captação de recursos e transferência de produtos e serviços relativos aos temas estratégicos 
"Reconhecimento institucional" e "Orientação para o mercado". Nessa unidade, os resultados com menores índices de desempenho se referem ao tema estratégico "Inovação e qualidade em P\&D", que contempla os OEs relacionados a projetos de P\&D focados no mercado, "Gerar e adaptar produtos e serviços de qualidade" e "Integração interestitucional".

\section{Acompanhamento de execução dos OEs da UD X/2002}

Temas do fliagrama

- Oricatacào para o mereado

- Inovaçân e qualídade em PAD

- Reconhecimento institucional

- Exceléncia cm gestão institucional
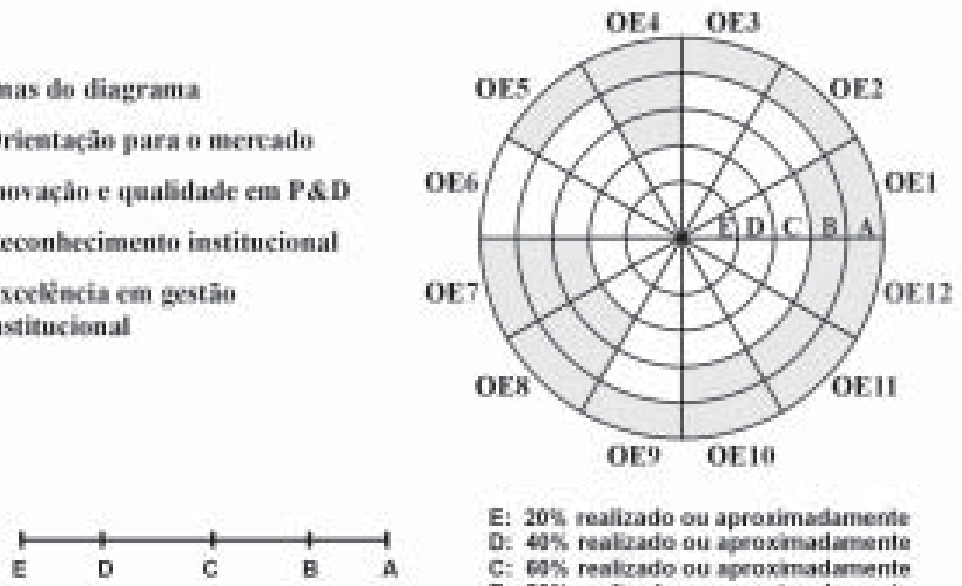

E: $20 \%$ realizado ou aproximadamente D. $40 \%$ realezado ou apeoximadamente C: $69 \%$ realizado ou apeoximadamente B: $80 \%$ realuado ou aproximadamente A: $100 \%$ realizado ou aproximadamente

Figura 8: Avaliação da implementação do MGE da UD X.

\section{Conclusões}

O Método MGE/Acompanha, nas versões 2000 e 2002, mostrouse efetivo para identificar as dificuldades e os benefícios advindos da introdução de processos e atividades estratégicas típicos da teoria da administração moderna, incrementados em parte na Embrapa, identificando, inclusive, o quanto do MGE foi possível implementar, para que se promovesse o alinhamento de indivíduos, equipes, unidades organizacionais e instrumentos gerenciais às estratégias corporativas, as quais também preconizam maior integração intra e interunidades e captação de recursos. O método revelou a deficiência de habilidade e conhecimento de alguns gerente de modelo e de objetivos estratégicos para desenvolver o pensamento estratégico no tempo e na velocidade que o ambiente externo requer. 
${ }^{1}$ Os pontos de vista expressos neste trabalho são advindos da compilação das respostas dos questionários respondidos pelos GOEs do MGE

\section{Referências bibliográficas}

Coutinho, A. Construindo uma organização focada na estratégia. In: Reunião DE ChEFES DA EmBRAPA, 2002, Planaltina. Palestra. Planaltina, DF: Symnetics, 2002.

EMBraPA. Departamento de Organização e Desenvolvimento. MGE/Acompanha: método de acompanhamento da implantação de Modelo de Gestão Estratégica; Versão 1.0. Brasília, DF, 2000, publicação interna. 20 p.

. Departamento de Organização e Desenvolvimento. Modelo de Gestão Estratégica: descrição geral. Brasília, DF, 2002.

Embrapa. Secretaria de Administração Estratégica. Mogest Simplificado - método para construção de Modelo de Gestão Estratégica, Versão 3.0. Brasília, DF, 2001. 40 p.

. Secretaria de Administração Estratégica. III Plano Diretor da Embrapa: realinhamento estratégico. 1999-2003. Brasília, DF, 1999. 36 p.

KaPLAn, Robert S.; Norton, David P. A estratégia em ação. 5. ed. Rio de Janeiro: Campus, 1997. 344 p.

KAPLAN, Robert S. The Balanced Scorecard. Washington: Harvard Business School Press, 1996. 344 p.

NoRTON, D. P. SFO Spider Charts: a time-phased process for managing strategic change. In: Balanced Scorecard North American Summit, 2001, San Diego. Apostila. San Diego: Balanced Scorecard Collaborative, 2001. 
Anexos

Anexo 1: Formulário de acompanhamento de $\mathrm{OE}$

Formulário de acompanhamento de $\mathrm{OE}$

\begin{tabular}{l|l}
\hline OE\# & <Título do OE> \\
\hline
\end{tabular}

1. Estado atual

2. Entraves e dificuldades encontradas

3. Dúvidas

4. Sugestões para melhorar do processo de implementação do MGE

Anexo 2: Formulário de consolidação da situação dos OEs

\begin{tabular}{l|l}
\hline UD: & \multicolumn{1}{|c}{ <Nome da UD> } \\
\hline 1. Entraves e dificuldades encontradas \\
\hline 2. Dúvidas \\
\hline 3. Sugestões para melhorar o processo de implementação do MGE \\
\hline
\end{tabular}

Anexo 3: Plano de melhoria do MGE

\begin{tabular}{l|l|l|l|l}
\hline Ações propostas & Entraves & Prioridade & Prazo & Quem \\
\hline 1 & & & & \\
\hline 1.1 & & & & \\
\hline 1.2 & & & & \\
\hline $1 . n$ & & & & \\
\hline 2 & & & & \\
\hline 2.1 & & & & \\
\hline 2.2 & & & & \\
\hline $2 . n$ & & & & \\
\hline M & & & & \\
\hline M.1 & & & & \\
\hline M.2 & & & & \\
\hline M.n & & & & \\
\hline
\end{tabular}


Anexo 4: Formulário de acompanhamento da implementação do MGE

\begin{tabular}{|c|c|c|c|c|c|c|}
\hline \multirow[b]{2}{*}{$\mathbf{N}^{\circ}$} & \multirow[b]{2}{*}{ Descrição do item } & \multicolumn{5}{|c|}{ Valor } \\
\hline & & 1 & 2 & 3 & 4 & 5 \\
\hline 1 & Motivação, envolvimento e comprometimento das pessoas & & & & & \\
\hline 1.1 & Chefias & & & & & \\
\hline 1.1 .1 & Participação em reuniões do MGE & & & & & \\
\hline 1.1 .2 & Realização da reunião mensal & & & & & \\
\hline 1.1 .3 & Resolução dos itens da agenda do chefe & & & & & \\
\hline 1.1.4 & Supervisão efetiva da implementação do modelo & & & & & \\
\hline 1.2 & Gerente do modelo & & & & & \\
\hline 1.2 .1 & $\begin{array}{l}\text { Produtividade e criatividade na condução do processo de } \\
\text { implementação }\end{array}$ & & & & & \\
\hline 1.2 .2 & Nível de organização do trabalho & & & & & \\
\hline 1.3 & Gerentes de OEs & & & & & \\
\hline 1.3 .1 & Participação nas reuniões do MGE & & & & & \\
\hline 1.3 .2 & Proposição de melhorias ao processo de implementação do OE & & & & & \\
\hline 1.4 & Empregados em geral & & & & & \\
\hline 2 & Divulgação e comunicação do MGE na UD & & & & & \\
\hline 2.1 & Eventos de divulgação realizados & & & & & \\
\hline 2.1 .1 & Palestras & & & & & \\
\hline 2.1 .2 & Reuniões & & & & & \\
\hline 2.1 .3 & Oficinas & & & & & \\
\hline 2.1 .4 & Gincanas & & & & & \\
\hline 2.2 & Instrumentos de comunicação utilizado & & & & & \\
\hline 2.2 .1 & Lista de discussão & & & & & \\
\hline 2.2 .2 & Avisos nos murais & & & & & \\
\hline 2.2 .3 & Home page implantada & & & & & \\
\hline 2.2 .4 & E-mails informativos & & & & & \\
\hline 2.2 .5 & Notas no jornal interno & & & & & \\
\hline 2.2 .6 & Cartazes, faixas & & & & & \\
\hline 3 & Implementação efetiva do MGE & & & & & \\
\hline 3.1 & Inclusão do PAE no PAT & & & & & \\
\hline 3.2 & Alocação das atividades do PAE no SAAD dos empregados & & & & & \\
\hline 3.3 & Integração das iniciativas/ações do PAE com o SEP & & & & & \\
\hline 3.4 & $\begin{array}{l}\text { Resultados alcançados pelas ações do PAE e dos valores } \\
\text { dos indicadores de desempenho. }\end{array}$ & & & & & \\
\hline 4 & Desenvolvimento de pessoas & & & & & \\
\hline 4.1 & Eventos de capacitação gerencial & & & & & \\
\hline 4.2 & Eventos de desenvolvimento dos aspectos comportamentais & & & & & \\
\hline 4.3 & Eventos de capacitação técnica & & & & & \\
\hline
\end{tabular}




\section{Balanced Scorecard (BSC) - uma visão metodológica para o acompanhamento de sua implementação}

Edla M. B. Lima, Maristela J. da Silva, Marlene Araújo e Cintia P. da Cunha

A Embrapa, na intenção de operacionalizar a gestão dos seus planos estratégicos (Plano Diretor da Embrapa - PDE e Planos Diretores das Unidades - PDUs) desenvolveu a metodologia Mogest para a implementação do seu Modelo de Gestão Estratégica (MGE), no âmbito corporativo e de suas 40 unidades de negócio, com base na modificação do método Balanced Scorecard - BSC, de Kaplan. Posteriormente, após a implementação dos MGEs, no período de 1999 até os dias atuais, inicia-se e desenvolve-se a fase de acompanhamento por meio da elaboração do método MGE/Acompanha.

Este trabalho aborda, de forma modificada, o uso do diagrama elaborado por Norton, apud Coutinho (2002), para acompanhar/avaliar a implementação do BSC para uma organização orientada à estratégia, inserido no método MGE/Acompanha (2000), elaborado pela Equipe MGE e aplicado em uma das unidades de negócio da Embrapa. Os resultados mostraram a eficiência do método e proporcionaram a internalização do conceito "painel de bordo", desenvolvido no software Oracle BSC (OBSC).

\section{Balanced Scorecard (BSC) - una visión metodológica para el acompañamiento a su implementación}

Edla M. B. Lima, Maristela J. da Silva, Marlene Araújo y Cintia P. da Cunha

Para acompañar la implementación de sus planes estratégicos (PDE - el Plan Estratégico Corporativo y PDUs - los Planes Estratégicos de sus Unidades de Negócios), Embrapa há desarrollado y ha implementado al Modelo de Gestión Estratégica (MGE), basado en el Balanced Scorecard (BSC), una versión con escopo corporativo y cuarenta versiones para cada una de sus cuarenta unidades de negocio (los centros de investigación). Después de la implementación de todos MGEs, en el período de 1999 a hoy, Embrapa ha estado desarrollando y ha aplicado un método de la continuación llamado MGE/Acompanha.

Este artículo enfoca, de una manera modificada, el uso del diagrama elaborado por Kaplan \& Norton, para seguimiento y evaluación de la implementación del BSC en una Organización Focalizada en la Estratégia. Este diagrama fue inserido en el metodo MGE/ Acompanha elaborado por el Equipe MGE/Embrapa y el fue testado in una de las unidades de negocios de Embrapa. Los resultados obtenidos mostraran la eficiencia del metodo y ayudaran en la internalización del concepto de "painel de bordo", el cual fue implementado com la utilización del software Oracle OBSC.

\section{Balanced Scorecard (BSC) - a methodological view to keep track of its implementation}

Edla M. B. Lima, Maristela J. da Silva, Marlene Araújo and Cintia P. da Cunha

In order to keep track of its strategic plans implementation (PDE - Corporate Strategic Plan and PDUs - Business Units Strategic Plans), Embrapa has developed and has implemented the Strategic Management Model (MGE), based on the Balanced Scorecard (BSC), one version coporatewide and forty versions of each of its forty business units (research centers). Later, after the implementation of all MGEs, in the period of 1999 to today, Embrapa has been developing and applying a follow-up method called MGE/ Acompanha.
Revista do

Serviço

Público

Ano 54

Número 3

Jul-Set 2003

Edla Maria

Bezerra Lima,

MSc em

Geologia de Engenharia e Ambiental;

Maristela Jesus

da Silva, BSc.

em Ciências

Sociais;

Marlene de

Araújo, BSc, em

Comunicação

Social; e

Cintia Pereira

da Cunha,

graduada em

Administração.

Todas são

servidoras da

Secretaria de

Gestão e

Estratégia

(SGE) da

Embrapa,

Brasília-DF.

Contato:

edla.lima@

embrapa.br

maristela.silva@ embrapa.br

marlene@

sede.embrapa.br

cintia@

$\underline{\text { sede.embrapa.br }}$ 
This paper approaches, in a modified way, the use of the diagram elaborated by Kaplan \& Norton, to follow up and to evaluate BSC's implementation in a Strategyfocused Organization. This diagram has been inserted in the method MGE/Acompanha (2000) elaborated by the Team MGE/Embrapa and it was tested in one of Embrapas's business units of Embrapa. The results showed the efficiency of the method and they provided the absorption of the concept of "board panel", implemented using the software Oracle OBSC. 